\title{
Adverse drug reactions reported to a provincial public health sector pharmacovigilance programme in South Africa
}

\author{
J Jones, ${ }^{1}$ BPharm; A Swart, ${ }^{1}$ BSc Pharm; E Tommy, ${ }^{1}$ BPharm; K Cohen, ${ }^{2}$ MB ChB, MMed (Clin Pharm), MSc (Epidemiol), \\ Dip HIV Man (SA), Dip Obst (SA); A Stewart, ${ }^{3} \mathrm{MPH}$ (Epidemiol); J Voget, ${ }^{4}$ BPharm, MPhil (HIV Management); \\ M Blockman, ${ }^{2}$ BPharm, MB ChB, PG Dip Int Res Ethics, MMed (Clin Pharm)
}

\author{
${ }^{1}$ Medicines Information Centre, Division of Clinical Pharmacology, Department of Medicine, Faculty of Health Sciences, University of Cape Town, \\ South Africa \\ ${ }^{2}$ Division of Clinical Pharmacology, Department of Medicine, Faculty of Health Sciences, University of Cape Town, South Africa \\ ${ }^{3}$ UCT Clinical Research Centre, Cape Town, South Africa \\ ${ }^{4}$ Health Programmes, Department of Health, Western Cape Government, Cape Town, South Africa
}

Corresponding author: M Blockman (marc.blockman@uct.ac.za)

\begin{abstract}
Background. There are limited data in South Africa (SA) on adverse drug reaction (ADR) patterns and common causative medicines, outside of HIV and tuberculosis treatment programmes. In SA, Western Cape Province has a pharmacovigilance programme that collects spontaneous reports of suspected ADRs from public sector healthcare facilities.

Objectives. To describe reports received by the pharmacovigilance programme over a 4-year period (excluding those ascribed to medicines used to treat HIV and tuberculosis), as well as challenges faced in the implementation of such a system.

Methods. Reports of suspected ADRs and deaths possibly related to ADRs received between January 2015 and December 2018 were reviewed. Causality was assessed by a pharmacist, with multidisciplinary team involvement for all deaths and complicated cases. Causality was categorised according to the World Health Organization-Uppsala Monitoring Centre system. Preventability was assessed using Schumock and Thornton criteria. Observations on preventability and challenges faced in the operation of a spontaneous reporting system were also noted.

Results. We received 5346 reports containing 6023 suspected ADRs. There were 5486 ADRs confirmed after causality assessment, in 5103 reports. Cough, angio-oedema, movement disorders and uterine bleeding disorders were the most common ADRs. Enalapril, etonogestrel, amlodipine and hydrochlorothiazide were the most commonly implicated drugs. Seven deaths were reported; 3 of these reports of deaths had confirmed ADRs, and these ADRs were assessed as contributing to the deaths. Approximately $3.8 \%$ of commonly reported ADRs were preventable.

Conclusions. Enalapril and etonogestrel were responsible for a significant proportion of ADRs reported to this provincial programme. Future work should include quantification of preventability aspects to better inform gaps in healthcare worker knowledge that can be addressed in order to improve patient care.
\end{abstract}

S Afr Med J 2020;110(12):1226-1230. https://doi.org/10.7196/SAMJ.2020.v110i12.14721

The concept of pharmacovigilance and the need for the monitoring of medicine-related harms has been in existence for more than 150 years. ${ }^{[1]}$ The thalidomide limb malformation disaster in the 1960 s prompted the development of a number of spontaneous adverse event reporting systems and legislation in Europe and elsewhere, in order to regulate and monitor medicine safety.

The simplest form of pharmacovigilance involves spontaneous reporting systems that collect information on suspected adverse effects of drugs post marketing. ${ }^{[2]}$ The primary aim of a spontaneous reporting system is to detect early signals of new, serious or rare adverse drug reactions (ADRs). ${ }^{[2]}$ Pharmacovigilance is increasingly recognised and promoted as an important component of public health systems in order to support safe, rational and cost-effective use of medicines and to minimise any related harms. ${ }^{[3]}$

In Western Cape Province, South Africa (SA), the Western Cape Department of Health, in collaboration with the Medicines Information Centre (MIC) in the Division of Clinical Pharmacology at the University of Cape Town (UCT), introduced a targeted spontaneous reporting system for suspected ADRs to antiretrovirals in 2005, to inform the antiretroviral treatment programme regarding the safety of medicines used in the programme. ${ }^{[4]}$ This programmatic pharmacovigilance system, which also included tuberculosis treatment from 2012, has been described previously. ${ }^{[4]}$ In 2014, this service was further expanded to provide a broader pharmacovigilance service that solicits reports of suspected ADRs to all medicines encountered in Western Cape public sector healthcare facilities. The goals of this reporting system are to identify possible signals of previously undocumented rare ADRs, or increased severity of known ADRs, and to instil a culture of drug safety awareness among healthcare workers (HCWs). The programme encourages HCWs to report all suspected ADRs, especially serious reactions that are potentially life-threatening, cause hospitalisation or extended hospital stay, or permanent disability, or require the offending drug to be stopped. In addition, any deaths suspected to be associated with an ADR and any exposure to a known teratogen are also requested to be reported. Quarterly and annual reports are shared with HCWs, including preventability issues and drug safety learning points.

\section{Objectives}

We describe reports to the Western Cape pharmacovigilance service over a 4-year period between January 2015 and December 2018, excluding suspected ADRs to HIV and TB medications, which 
are described elsewhere. ${ }^{[4]}$ We also describe lessons learned and challenges faced in the implementation of an ADR reporting system in a public health system environment.

\section{Methods}

ADR reports were submitted to the MIC by HCWs using a standardised reporting form, which is also used by the national medicines regulatory authority. Reports could be submitted by email or fax, and since June 2016 reports could be submitted electronically via Sinjani, which is the reporting portal for public sector facilities in the Western Cape. Reports are stored in a registry maintained by the MIC. Initial assessment of the reports is performed by a pharmacist, who contacts the reporter for additional information should it be required for assessment of causality and preventability.

We reviewed reports received between January 2015 and December 2018. An information pharmacist performed causality assessment of suspected ADRs. A clinical pharmacologist and/or a doctor was included in causality assessment for complex cases and deaths. We used the World Health Organization-Uppsala Monitoring Centre (WHO-UMC) system ${ }^{[5]}$ for assessing causality, which classifies ADRs as definite, probable, possible, unlikely or unassessable.

'Confirmed ADRs' were those categorised as definite, possible or probable using the WHO-UMC system. ${ }^{[5]}$ For all deaths, the contribution of any confirmed ADR to the death was assessed, and the ADR was classified as a major contributor, contributor or noncontributor to the patient's death.

MedDRA terminology was used to describe the ADRs. Individual ADRs were also grouped under a higher-level term describing an ADR category, as set out in Table 1. MedDRA is a hierarchical medical terminology dictionary used by pharmaceutical industry regulators for recording of drug safety data, among other things. ${ }^{[6]}$

Schumock and Thornton criteria ${ }^{[7]}$ were used to assess preventability. The ADR was categorised as preventable if any of the following criteria were met: drug prescribed inappropriate, dose/ route/frequency inappropriate, laboratory monitoring not performed as required, history of a previous reaction to the drug, drug interaction, toxic serum drug level, and poor compliance.

The MIC maintains a repository of ADRs reported to the Western Cape pharmacovigilance system with UCT Human Research Ethics Committee (HREC) approval (ref. no. R040/2016). This sub-study also has UCT HREC approval (ref. no. 541/2019).

\section{Results}

From 1 January 2015 to 31 December 2018, we received 5346 reports detailing 6023 suspected ADRs. After causality assessment, there were 5486 confirmed ADRS, which were detailed in 5103 reports. Patient characteristics for these 5103 reports, as well as the cadre of HCW submitting the report, are described in Table 2.

Causality according to the WHO-UMC system was assessed as follows: certain $n=142$ ( $n=2$ with multiple suspect drugs), probable $n=1906$ ( $n=81$ with multiple suspect drugs), and possible $n=3438$ ( $n=84$ with multiple suspect drugs). Two suspected ADRs were assessed as unlikely to be an ADR, and 535 ADRs were unassessable.

The 10 most commonly reported ADR categories as well as the top implicated drug are reflected in Fig. 1. The 10 most commonly implicated drugs and the associated ADR categories are presented in Table 3.

Seventy-six percent of ADRs (4 132/5 435) were in the top 10 ADR categories. In the top 10 ADR categories, 159 ADRs were

\begin{tabular}{ll} 
Table 2. Patient and reporter characteristics & \\
\hline & $\boldsymbol{n}(\%)$ \\
\hline Patient characteristics $(N=5103)$ & \\
$\quad$ Sex & $3829(75.0)$ \\
$\quad$ Female & $1226(24.0)$ \\
Male & $48(0.9)$ \\
Unknown & \\
Age & $4793(93.9)$ \\
Adults and adolescents $>12$ years & $61(1.2)$ \\
$\quad \leq 12$ years & $249(4.9)$ \\
Unknown & \\
Number of confirmed ADRs in the report & $4827(94.6)$ \\
1 & $233(4.6)$ \\
2 & $43(0.8)$ \\
3 - 5 & \\
Reporter characteristics & $2621(51.4)$ \\
Doctor & $1255(24.6)$ \\
Nurse & $987(19.3)$ \\
Pharmacist & $150(2.9)$ \\
Other & $89(1.7)$ \\
Unknown & \\
ADRs = adverse drug reactions. &
\end{tabular}

Table 1. ADR categories and terms

\begin{tabular}{ll}
\hline ADR category & ADR terms \\
\hline Rashes, eruptions and exanthems NEC & Rash NOS, Rash erythematous, Rash papular, Rash maculo-papular, Rash macular, \\
& Rash morbilliform, Rash generalised \\
Muscle disorders & Muscle cramps, Myalgia, Muscle rupture, Rigors \\
General system disorders NEC & Oedema, Condition aggravated \\
Nausea and vomiting symptoms & Nausea, Nausea and vomiting, Nausea, vomiting and diarrhoea \\
Neurological disorders NEC & Dizziness, Ataxia, Tics \\
Headaches NEC & Headache \\
Menstrual cycle and uterine bleeding disorders & Menorrhagia, Metrorrhagia, Dysfunctional uterine bleeding, Menstruation irregular, \\
& Amenorrhoea, Dysmenorrhoea \\
Movement disorders & Dystonia, Extrapyramidal disorder NOS, Tremor, Parkinsonism, Akathisia, Tardive \\
& dyskinesia, Neuroleptic malignant syndrome, Oculogyric crisis, Torticollis, Rabbit \\
Angio-oedemas & syndrome \\
Coughing and associated symptoms & Angio-oedema, Swollen tongue, Lip swelling \\
ADR = adverse drug reaction; NEC = not elsewhere classified; NOS = no other symptoms.
\end{tabular}




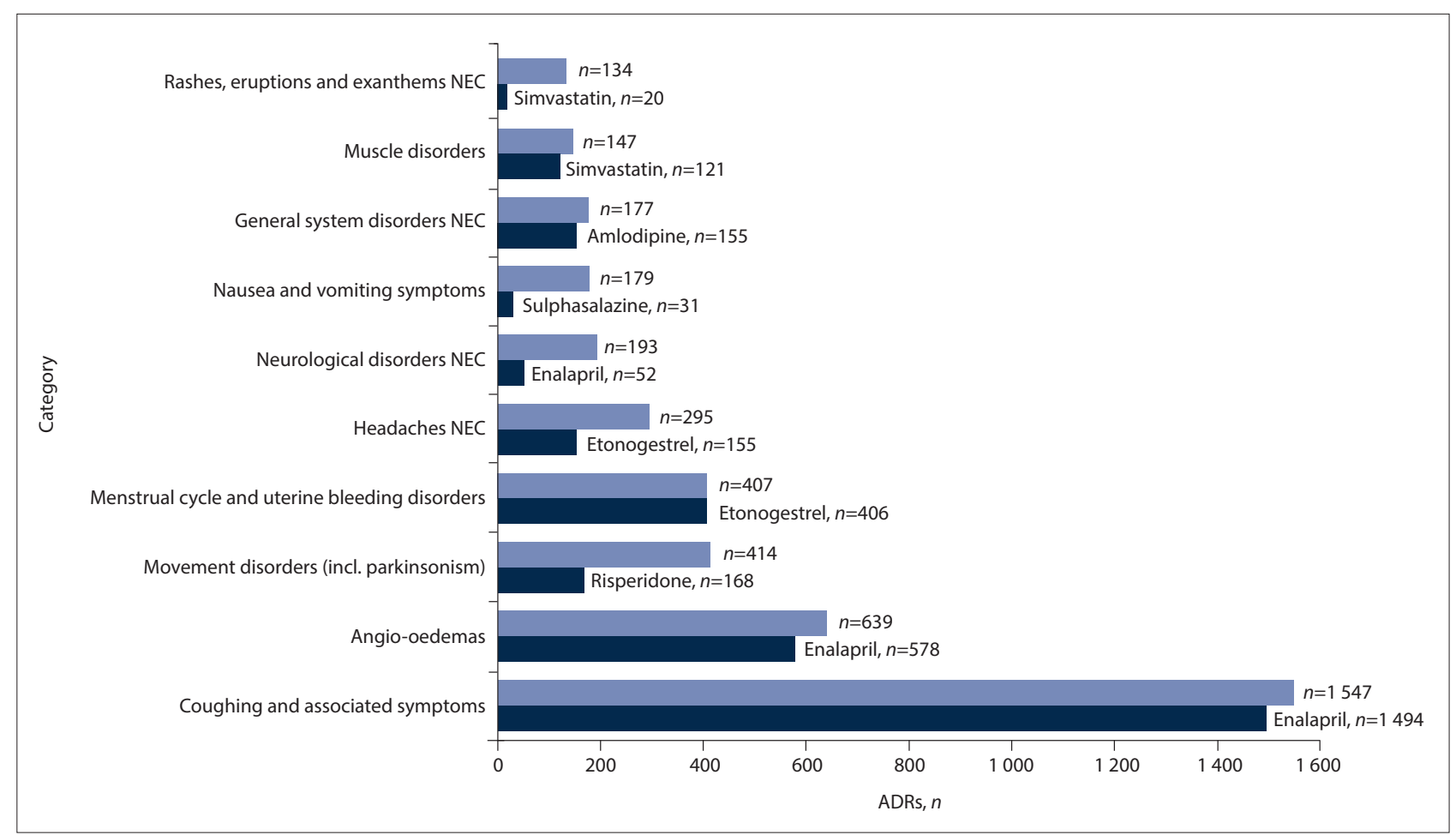

Fig. 1. Top 10 ADR categories and top implicated drug in each category, $2015-2018 .(A D R=$ adverse drug reaction; NEC = not elsewhere classified.)

\begin{tabular}{|c|c|c|}
\hline Implicated drug & Reports, $n$ & Top 3 associated ADR categories \\
\hline Enalapril & 2337 & $\begin{array}{l}\text { Coughing and associated symptoms }(n=1494 ; 63 \%) \text {, Angio-oedemas }(n=578 ; 25 \%) \text {, } \\
\text { Headaches NEC }(n=64 ; 3 \%)\end{array}$ \\
\hline Etonogestrel & 816 & $\begin{array}{l}\text { Menstrual cycle and uterine bleeding disorders }(n=406 ; 50 \%) \text {, Headaches NEC }(n=155 ; 19 \%) \text {, } \\
\text { Appetite and general nutritional disorders }(n=73 ; 9 \%)\end{array}$ \\
\hline Amlodipine & 567 & $\begin{array}{l}\text { General system disorders NEC }(n=155 ; 27 \%) \text {, Coughing and associated symptoms }(n=151 \text {; } \\
27 \%) \text {, Angio-oedemas }(n=72 ; 13 \%)\end{array}$ \\
\hline Hydrochlorothiazide & 409 & $\begin{array}{l}\text { Coughing and associated symptoms ( } n=189 ; 46 \%) \text {, Angio-oedemas }(n=96 ; 23 \%) \text {, } \\
\text { Muscle disorders }(n=18 ; 4 \%)\end{array}$ \\
\hline Simvastatin & 356 & $\begin{array}{l}\text { Muscle disorders ( } n=121 ; 34 \%) \text {, Coughing and associated symptoms ( } n=79 ; 22 \%) \text {, } \\
\text { Angio-oedemas }(n=29 ; 8 \%)\end{array}$ \\
\hline Risperidone & 305 & $\begin{array}{l}\text { Movement disorders including parkinsonism ( } n=168 ; 55 \%) \text {, Breast disorders }(n=69 ; 23 \%) \text {, } \\
\text { Salivary gland conditions }(n=24 ; 8 \%)\end{array}$ \\
\hline Atenolol & 179 & $\begin{array}{l}\text { Coughing and associated symptoms }(n=58 ; 32 \%) \text {, Neurological disorders NEC }(n=15 ; 8 \%) \text {, } \\
\text { Angio-oedemas }(n=12 ; 7 \%)\end{array}$ \\
\hline Metformin & 170 & $\begin{array}{l}\text { Gastrointestinal motility and defaecation disorders }(n=46 ; 27 \%) \text {, Coughing and associated } \\
\text { symptoms }(n=34 ; 20 \%) \text {, Nausea and vomiting symptoms }(n=20 ; 12 \%)\end{array}$ \\
\hline Haloperidol & 120 & $\begin{array}{l}\text { Movement disorders including parkinsonism }(n=106 ; 88 \%) \text {, Breast disorders }(n=5 ; 4 \%) \text {, } \\
\text { Salivary gland conditions }(n=4 ; 3 \%)\end{array}$ \\
\hline Sodium valproate & 119 & $\begin{array}{l}\text { Movement disorders including parkinsonism }(n=64 ; 54 \%) \text {, Salivary gland conditions } \\
(n=19 ; 16 \%) \text {, Platelet disorders }(n=7 ; 6 \%)\end{array}$ \\
\hline
\end{tabular}

assessed as preventable (159/4 132; 3.8\%). Some common themes in terms of preventability were observed. These included: (i) recurrent episodes of persistent cough due to rechallenges of enalapril (in some cases this was mismanaged by switching to a different brand of enalapril); and (ii) recurrent episodes of angio-oedema while remaining on enalapril, or due to inadvertent rechallenges. There were also 3 reports of menstrual cycle and uterine bleeding disorders and 1 of contraceptive failure in patients with etonogestrel implants who were on concomitant enzyme-inducing medications (efavirenz, phenytoin). These drug interactions may have resulted in decreased etonogestrel concentrations, with resultant contraceptive failure.

We received 21 reports of suspected ADRs in pregnant women, of which we confirmed 21. There were 3 cases of anaphylaxis due to cefazolin, 2 cases of angio-oedema due to enalapril (which is also contraindicated in pregnancy, as are all angiotensin-converting enzyme (ACE) inhibitors ${ }^{[8]}$ ), and 16 cases of inadequate spinal anaesthesia for caesarean section using bupivacaine. The cases of inadequate spinal anaesthesia despite correct administration pointed 
to a possible product quality issue that was referred to the relevant authority for further investigation.

Seven reports of deaths in which an ADR was suspected were received during the period under review. Four of these deaths were unassessable owing to a paucity of clinical information, despite repeated attempts to contact the reporters. The details of the remaining three deaths were as follows:

A 59-year-old woman with hypertension and breast cancer was receiving paclitaxel, dexamethasone, promethazine, cimetidine, amlodipine and hydrochlorothiazide. She developed angiooedema during a paclitaxel infusion and subsequently died despite interventions to intubate and resuscitate. This episode was assessed as a certain ADR attributed to paclitaxel and was a major contributor to the patient's death.

An 11-month-old female infant with bilateral pneumonia and pulmonary hypertension was being treated with amikacin, piperacillin/tazobactam and cloxacillin. She developed acute renal failure and subsequently died. It transpired that she had been given amikacin 8-hourly instead of once daily. The amikacin level was measured and found to be in the toxic range. This death was assessed as a probable and preventable ADR that was a major contributor to the patient's death.

A 42-year-old known hypertensive and diabetic patient suffered an inferolateral myocardial infarction. He was given 1.5 MU streptokinase (no contraindications) as per protocol and subsequently developed eight subarachnoid bleeds and died. He also received enoxaparin, aspirin, clopidogrel, simvastatin and insulin. The streptokinase was assessed as a probable cause of subarachnoid haemorrhage, with enoxaparin, aspirin and clopidogrel as other possible contributors. Subarachnoid haemorrhage was a major contributor to the patient's death.

\section{Discussion}

Over a 4-year period, the MIC (on behalf of the Western Cape Province pharmacovigilance programme) received 5346 reports of suspected ADRs, excluding reports where HIV and/or TB medications were implicated. Of 6023 suspected ADRs, 5486 were confirmed on causality assessment, of which $3.8 \%$ were assessed as preventable. This is low compared with a recent prospective study in our setting. ${ }^{[9]}$ The most common ADRs reported were cough, angio-oedema, movement disorders and menstrual/uterine bleeding disorders, which corresponds to the known ADR profiles of the medications in the standardised formularies in SA, which include the Essential Medicines List (EML) and the Practical Approach to Care Kit (PACK). ${ }^{[10]}$ Three patients died with an ADR considered to have contributed directly to the death.

Approximately $41 \%$ of all confirmed ADRs were associated with the use of enalapril, of which cough and angio-oedema were the most common. In 2015, access to the angiotensin receptor blocker losartan was expanded to allow medical officers to prescribe it in consultation with a specialist, provided an ADR form reporting enalapril intolerance was also completed. This policy is likely to have contributed to the high proportion of reported ADRs that implicated enalapril. Many cases of cough were assessed as preventable due to subsequent re-initiation of the offending drug. This re-initiation may have been due to insufficient record-keeping. In some cases, the cough was mismanaged by switching to a different brand, highlighting the need for education of HCWs about the aetiology of ACE inhibitor-induced cough. Some cases of angio-oedema were categorised as preventable because of repeated episodes in the same patient before the offending drug was stopped. These recurrent episodes emphasise the importance of questioning patients about the occurrence of side-effects at regular intervals, and also of having systems in place to alert other HCWs to patients with known allergies (e.g. electronic health records, stickers on folders and MedicAlert bracelets). These issues were communicated back to HCWs via the quarterly and annual reports, and a poster is also being developed summarising angio-oedema and its management.

The second most commonly implicated drug was the etonogestrel implant. This contraceptive option was introduced into the public sector formulary in November 2013 and actively promoted as a preferred choice. The large number of ADR reports may suggest some patient dissatisfaction with this option, or alternatively increased ADR awareness and vigilance on the part of HCWs after the introduction of a new medication.

\section{Study limitations and strengths}

The main limitation of this study is that ADRs are probably underreported, which is a known weakness of all spontaneous reporting systems. We cannot calculate the incidence or prevalence of ADRs or identify risk factors, as we do not have denominator data. ${ }^{[3]}$ Causality assessment was often challenging owing to provision of insufficient information. Another limitation is that repeated entries for individual patients are not linked in the database, so there may be repeated ADR reports submitted for some individuals. A strength of this study is the standardised, widely used causality assessment by a pharmacist, with multidisciplinary team involvement in assessment of more complex cases and all deaths. Future research could incorporate quantitative measurement of preventable ADRs in order to better identify gaps in HCW knowledge that can be addressed.

In an effort to make reporting of ADRs easier, an internet/intranetbased option was added to the paper-based reporting form in the Western Cape's electronic health information system, Sinjani. The actual design and building of the ADR module was challenging in terms of communicating requirements and desired functionality to the IT developers. Once this module was operational, it was discovered that many reports were being captured retrospectively by non-medical data capturers, which often resulted in incorrectly entered information due to factors such as illegible handwriting and insufficient medical and pharmaceutical knowledge. Extra followup was therefore needed by the pharmacist assessing causality. In addition, it was also often difficult to trace the original reporter. A number of communications had to be sent out requesting that only HCWs personally submit reports, but with time this has resolved, and the Sinjani option is a useful addition to methods of reporting. Another challenge has been difficulty in assessing causality due to provision of insufficient information. In this cohort, 535/6 023 suspected ADRs (8.9\%) were unassessable according to WHO criteria. Examples of missing information include no start/ stop dates of medicines; lack of information on dose, frequency of administration and duration of therapy for each medicine; lack of information on date of onset, duration, resolution and outcomes of reaction; insufficient supporting information on the type of reaction (e.g. renal impairment described but without any laboratory results); and only listing the suspected drug and not concomitant medications. This problem can be overcome to some extent by having mandatory fields in the electronic form, but it is still an issue with the paperbased forms.

\section{Conclusions}

Enalapril followed by etonogestrel were the most commonly implicated medicines and responsible for a significant proportion 
of ADRs reported to this provincial pharmacovigilance programme. Many enalapril ADRs could have been prevented by appropriate attention to the patient's history and accessible drug information.

In addition, the programme highlighted product quality issues that resulted in treatment failure in some patients.

This spontaneous voluntary reporting system in a public health sector environment provides useful information regarding the types of ADRs experienced and underscores the importance of accessible and visible patient records. It highlights medicine-related gaps in knowledge of healthcare workers. Guidance on diagnosis, prevention and management of these ADRs should be prioritised for inclusion in HCW training in order to improve patient care. It may also reinforce a culture of drug safety awareness and the avoidance of medicinerelated harms.

\section{Declaration. None.}

Acknowledgements. We thank all HCW colleagues who reported ADRs to the pharmacovigilance programme.

Author contributions. MB conceived the study; ET and JJ collated the data; ET, KC and MB comprised the multidisciplinary team for causality assessment; ASt analysed the data; JJ and MB prepared the first draft of the manuscript; ASt, ASw, ET, KC and JV reviewed the manuscript and gave input. All authors read and approved the final manuscript. Investigators from the Division of Clinical Pharmacology were responsible for study design, data analysis and data interpretation.
Funding. The Western Cape Pharmacovigilance Programme is an ongoing collaboration between the MIC, Division of Clinical Pharmacology, UCT, and the Western Cape Department of Health. The pharmacovigilance programme is funded by the Western Cape Department of Health. Conflicts of interest. None.

1. Routledge P. 150 years of pharmacovigilance. Lancet 1998;351(9110):1200-1201. https://doi org/10.1016/S0140-6736(98)03148-1

2. Harmark L, van Grootheest AC. Pharmacovigilance: Methods, recent developments and future perspectives. Eur J Clin Pharmacol 2008;64(8):743-752. https://doi.org/10.1007/s00228-008-0475-9

3. World Health Organization. The safety of medicines in public health programmes: Pharmacovigilance an essential tool. 29 October 2019).

4. Cohen K, Jones I, Stewart A, et al. Adverse drug reactions reported to the Western Cape programmatic pharmacovigilance programme in South Africa: 2007 to 2017. Pharmacoepidemiol Drug Saf pharmacovigilance programme in South Africa:
2018;27(S2):340. https://doi.org/10.1002/pds.4629

5. Uppsala Monitoring Centre. The use of the WHO-UMC system for standardised case causality 5ppsala Monitoring Centre. The use of the WHO-UMC system for standardised case causality
assessment. https://www.who.int/medicines/areas/quality_safety/safety_efficacy/WHOcausality_ assessment. https://www. who.int/medicin
assessment.pdf (accessed 14 November 2019).

6. MedDRA: Medical Dictionary for Regulatory Activities. https://www.meddra.org/basics (accessed 14 November 2019)

Schumock G, Thornton J. Focusing on the preventability of adverse drug reactions. Hosp Pharm 1992;27(6):538.

8. August P. Adverse effects of angiotensin converting enzyme inhibitors and receptor blockers in pregnancy. UpToDate, last updated 10 December 2019. https://www.uptodate.com/contents/ adverse-effects-of-angiotensin-converting-enzyme-inhibitors-and-receptor-blockers-in pregnancy?search=angiotensin-converting $\% 20$ enzyme $\% 20$ inhibitors $\% 20$ pregnancy\&source=search result\&selectedTitle $=2 \sim 127$ \&usage_type=default\&display_rank=1 (accessed 11 December 2019).

9. Mouton JP, Njuguna C, Kramer N, et al. Adverse drug reactions causing admission to medical wards A cross-sectional survey at 4 hospitals in South Africa. Medicine (Baltimore) 2016;95(19):e3437 https://doi.org/10.1097/MD.0000000000003437

10. Knowledge Translation Unit, University of Cape Town Lung Institute. Practical Approach to Care Kit. https://knowledgetranslation.co.za/pack/wc-south-africa/ (accessed 11 December 2019).

Accepted 18 August 2020 